\title{
Development of a thermo-hydraulic bypass leakage test method for the Wendelstein 7-X target element cooling structure
}

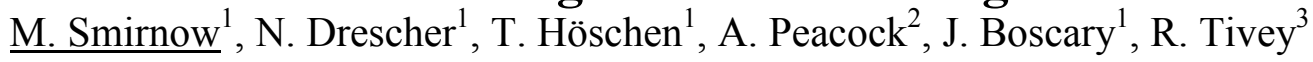 \\ ${ }^{1}$ Max-Planck-Institut für Plasmaphysik, EURATOM Association, 85748 Garching, Germany \\ ${ }^{2}$ European Commission c/o Max-Planck-Institut für Plasmaphysik, EURATOM Association, 85748 Garching, Germany \\ ${ }^{3}$ European Commission clo EFDA JET, Culham Science Centre, Abingdon, Oxfordshire, OX14 3DB, United Kingdom
}

A facility for testing the cooling structure to ensure the quality of the target element heat sink is under construction at IPP Garching. This test bed has been built up to do a proof of concept study with a hydraulic mock up, built from the same material as the target elements, but can be opened for artificial gap manufacture.

A bypass in the cooling structure was the main reason for the overheating of CFC tiles, which resulted in a defect by cracking of the CFC - AMC-Cu interlayer in pre-series 3. The cooling structure is built from two half-shells, in both a half pipe is milled [1]. To correlate the thermal response function to the artificial bypass gaps the time constant of the cool down was used. The results of the measurements are presented and compared with the calculated results.

Keywords: Wendelstein 7-X, Divertor, Target element, Cooling structure, Bypass, Thermohydraulic Flow Test

\section{Introduction}

The $\mathrm{CuCrZr}$ cooling structure of the target elements is manufactured by electron beam welding together two half plates into which semi-circular channels are machined. This fabrication route can allow the possible opening of gaps between adjacent channels due to manufacturing tolerances. To minimize the occurrence of bypasses two design changes have been implemented to the fabrication route. They should minimize the effect of bypass leakage inside the cooling structure, nevertheless the extreme influence of this effect on the cooling performance and life time of target elements gives the motivation to develop a practicable test method which ensures the hydraulic quality of the target element heat sink.

The size of the gap and the consequent leakage has a significant impact on the performance of the elements, especially for the longest type of elements like $1 \mathrm{~S}(\sim 600 \mathrm{~mm})$. It results in a reduced local flow velocity at the end of the element that decreases the critical heat flux margin and also the heat transfer capability that will induce higher temperature in the critical interface between CFC tiles and copper.

Based on calculations, it should be feasible to identify gaps with $>75 \mu \mathrm{m}$ width through pressure drop flow tests and thermal transient response tests. In order to study this effect, a test bed has been built up at IPP to perform a proof of concept study with a hydraulic mock up. It is built from the same $\mathrm{CuCrZr}$ material as the target elements, and is similar in shape and weight but with a lid that can be opened to allow the artificial gap to be adjusted.

The purpose is to measure the transient thermal response of the element subjected to a warm/cold water front. The delayed response of thermocouples is related to the local heat transfer coefficient at the wall. It characterizes the local flow velocity, which is related to possible leakage and gap size.
This document presents the thermo-hydraulic tests performed at IPP Garching with the hydraulic mock up and with a $1 \mathrm{~S}$ pre-series element with the optimized cooling structure design.

\subsection{Calculated effect of a bypass to the cooling performance}

If a bypass between adjacent cooling channels exists, the cooling water flows partially through the bypass, and thus the flow through the main channel is reduced which causes a lower pressure drop.

Because the overall inlet flow is constant, the coolant velocity in the cooling tube decreases.

Fig. 1 shows the calculated loss of coolant velocity in case of a bypass between adjacent cooling channels based on R. Tiveys work [2].

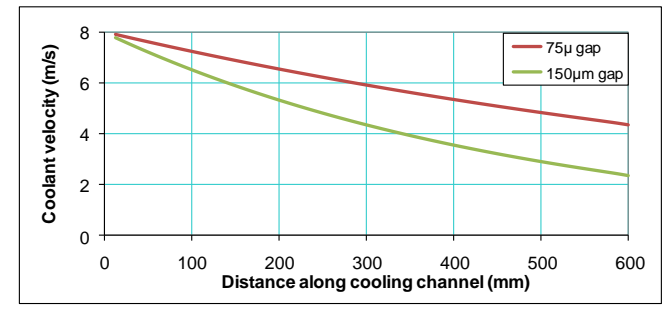

Fig.1 Drop of coolant velocity along the target element for $75 \mu$ and $150 \mu \mathrm{m}$ gaps.

A $75 \mu \mathrm{m}$ gap over the length of a $600 \mathrm{~mm}$ long target element (type 1S) reduces the coolant velocity from $8 \mathrm{~m} / \mathrm{s}$ to $4 \mathrm{~m} / \mathrm{s}$ at the end of the target element. A decreasing coolant velocity reduces the heat transfer coefficient between coolant and wall. The heat transfer coefficient is calculated with Gnielinski's correlation:

$$
N u=\frac{\frac{\xi}{8}(R e-1000) P r}{1+12,7 \sqrt{\frac{\xi}{8}}\left(\operatorname{Pr}^{2 / 3}-1\right)} \cdot K_{P r}
$$

With,

$$
\alpha=N u \frac{\lambda}{D_{H}} \text { (2) and } \operatorname{Re}=\frac{\omega_{\text {swirl }} D_{H}}{v} \text { (3) }
$$


where $\omega$ represents the fluid velocity and $D_{H}$ the cooling tube diameter. The factor $\mathrm{K}_{\mathrm{Pr}}$ takes the temperature dependence of waters Prandtl number into account. Inside the target element swirl tapes with the twist ratio TR are placed as turbulence promoters, which leads to a higher coolant velocity $\omega_{\text {swirl }}$ relative to the wall:

$$
\omega_{\text {swirl }}=\omega \cdot\left(1+\frac{\pi^{2}}{4} T R^{2}\right)^{0,5}
$$

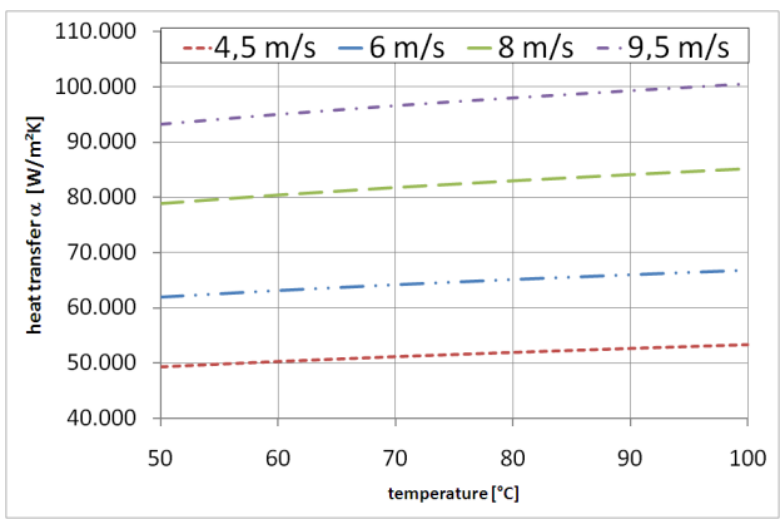

Fig.2 Heat transfer coefficient as a function of water temperature and coolant velocity

The calculated heat transfer coefficients shown in Fig. 2 have been used as input data for thermal transient finite element calculations with $A_{N S Y S}{ }^{\circledR}$ on a 3-D model of the middle part of a target element shown in Fig.3. In the test bed, the thermocouples are placed on the side of the target element, next to the cooling channels.

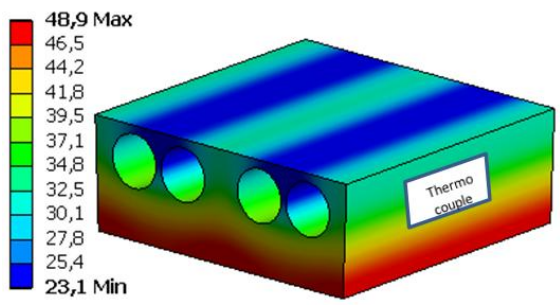

Fig. 3 Temperature distribution from $75^{\circ} \mathrm{C}$ to $20^{\circ} \mathrm{C}$ with a $4,5 \mathrm{~m} / \mathrm{s}$ convection after $0,9 \mathrm{~s}$ cooldown time.

The Ansys ${ }^{\circledR}$ simulations have been carried out in the range from $4,5 \mathrm{~m} / \mathrm{s}$ to $9,5 \mathrm{~m} / \mathrm{s}$ coolant velocity. The simulations results are extracted at the thermocouples position and then normalized as shown in Fig.4.

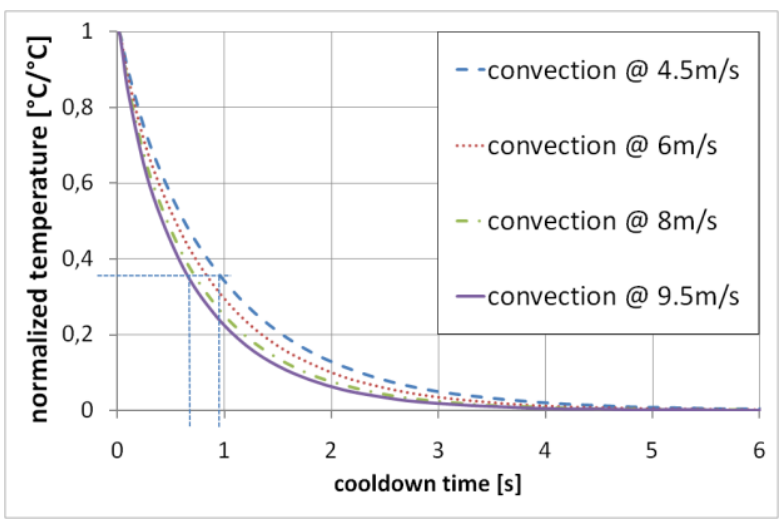

Fig.4 Cool down calculations from $75^{\circ} \mathrm{C}$ to $20^{\circ} \mathrm{C}$ with different convections.

In the simplest assumption for a very small and perfect attached thermocouple, the temperature development in time can be described analytically as a linear first order differential equation:

$$
C \frac{d T}{d t}=-\kappa\left(T-T_{0}\right)
$$

where $\mathrm{C}$ is the sample heat capacity and $\kappa$ is the thermal conductance of the heat sink. The solution of this differential equation is a first order exponential decay:

$$
T(t)=\Delta T \cdot e^{-\frac{t}{\tau}}-T_{0}(6) .
$$

The time constant,

$$
\tau=\frac{c \cdot m}{\kappa}
$$

consists of the specific heat capacity and the mass of $\mathrm{CuCrZr}$ around the thermocouple where heat is stored which affects the thermocouple. The thermal conductance of the target element

$$
\kappa=\frac{1}{\frac{l}{\lambda}+\frac{1}{\alpha}}
$$

includes the thermal conductivity $\lambda$ of $\mathrm{CuCrZr}$ and the temperature and coolant velocity dependent heat transfer coefficient $\alpha$. For an exponential decay, the time constant $\tau$ corresponds to a decay of temperature to $36,8 \%$ as shown in Fig. 5.

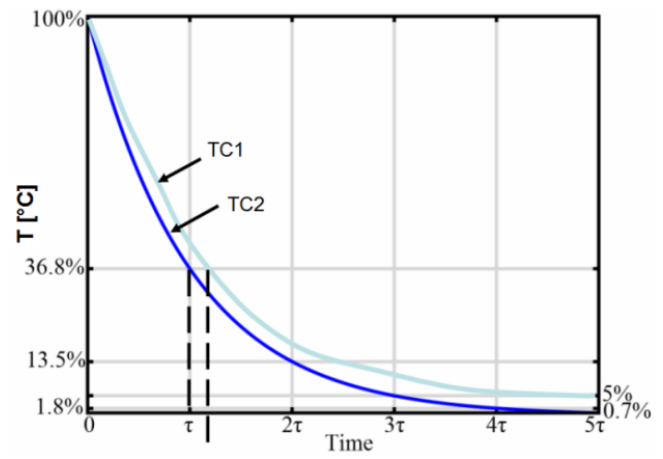

Fig.5 Shift of the time constant for two thermocouples.

Fig. 5 shows the expected time constant shift for two thermocouples placed in positions with a different heat transfer coefficient beneath.

If a $75 \mu \mathrm{m}$ gap is present over the whole length of the target element, the coolant velocity will drop (Fig.1) from $8 \mathrm{~m} / \mathrm{s}$ to $4 \mathrm{~m} / \mathrm{s}$ and thus the expected $\tau_{8 \mathrm{~m} / \mathrm{s}}$ $=0,6 \mathrm{~s}$ to $\tau_{4 \mathrm{~m} / \mathrm{s}}=0,9 \mathrm{~s}$ as shown in Fig. 4 .

\subsection{Calculated effect of a bypass to the pressure drop}

The effect of a gap between adjacent channels was estimated assuming a parallel gap. Following the 
design modification zero leakage is assumed between channels $2 \& 3$ and any by-pass flows at the inlet/outlet region or at the far end of the target plate remote from the inlet/outlet were neglected. The calculation was performed using an electrical analogy, to estimate the resistances of the various flow routes [2]. The calculation shows that a $75 \mu \mathrm{m}$ gap leads to a $12.5 \%$ change of pressure drop at $8 \mathrm{~m} / \mathrm{s}$. The pressure drop in a pipe between inlet pressure and outlet pressure is volume flow dependent,

$$
\Delta p=\left(\xi \frac{l}{d_{i}} \frac{\rho}{2}\right) w^{2}=p_{i}-p_{o}=k w^{2}
$$

thus, the $\mathrm{k}$-value can be determined from the slope of a linear regression of pressure drop measurement, at different flow rates. The k-value comprises the water density $\rho$, the length of the cooling tube 1 , the wall roughness and geometric aspects in $\zeta$.

\section{Test bed description}

The thermal shock test and the pressure drop measurement take place in the same test bed, shown in Fig.6. The use of the pump frequency to control the flow through the main water loop leads to distortions due to resonances inside the pump. In order to have a stable flow, two plug taps (V7 and V8) have been inserted into the main water loop as a bleeder chain.

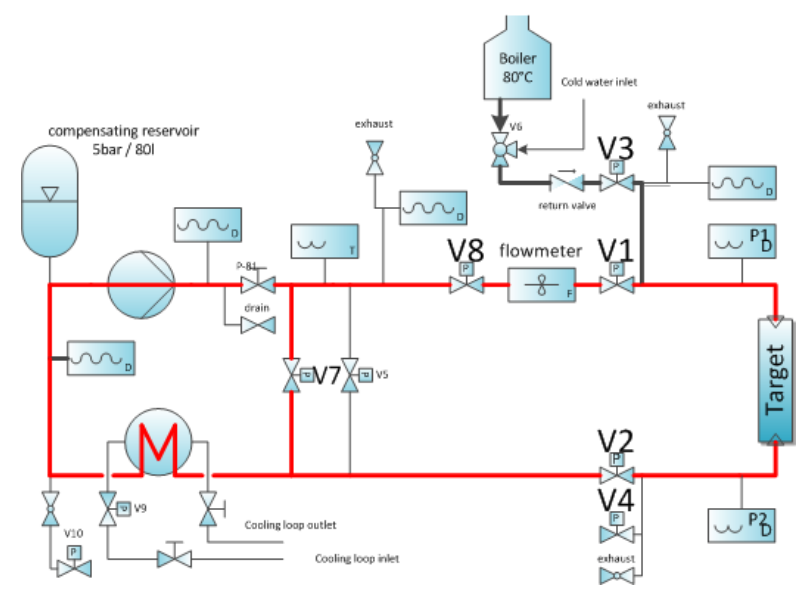

Fig. 6 The schematic of the thermal shock test bed shows the main water loop through the cooling structure and the flow control bypass in parallel in red.

By variation of the voltage of the plug taps V7 and V8, a flow operating map was created shown in Fig.7. The flow measurements showed that in a range of $1-2 \mathrm{~V}$ applied to the valve V7 it is possible to get a flow with a very low standard deviation by changing the voltage in V8 independent from the pump frequency.
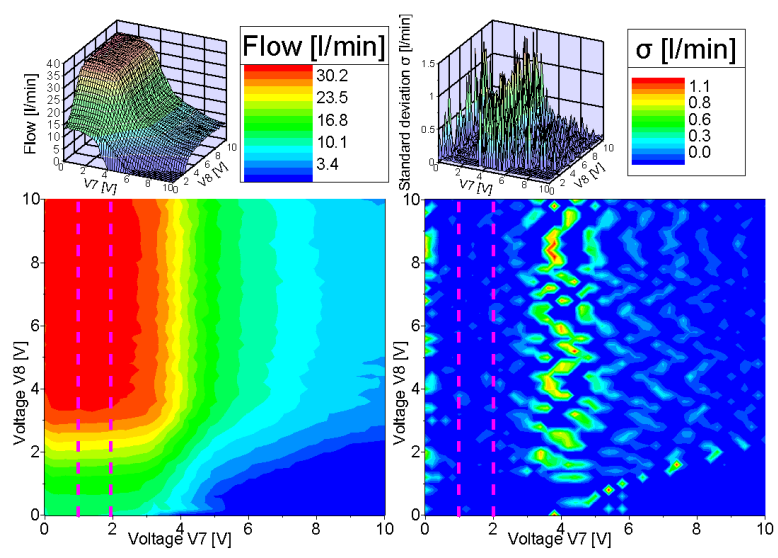

Fig.7 Flow operating map and standard deviation of the flow.

To set up a thermal shock test, the valves V1 and V2 are closed, and V3 and V4 opened. A boiler delivers $80^{\circ} \mathrm{C}$ hot water that flows over V3 into the cooling structure to V4 and then into a drain. When for all thermocouples a temperature around $75^{\circ} \mathrm{C}$ is reached, the valves $\mathrm{V} 3$ and $\mathrm{V} 4$ are closed, and $\mathrm{V} 1$ and V2 are synchronized opened. The cooling structure is equipped with 7 thermocouples sideways, and there are thermocouples placed in the inlet and outlet pipes of the cooling structure as shown in Fig.8.

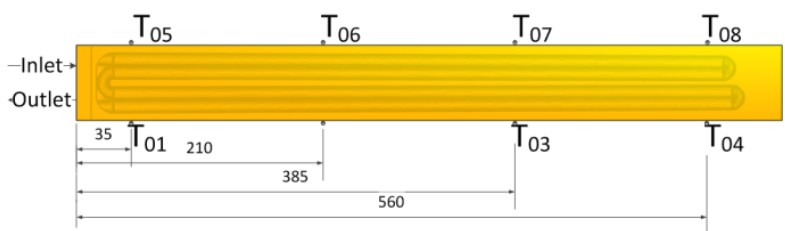

Fig. 8 Positions of the thermocouples attached to the hydraulic mock up.

For the test campaign, six different types of gaps have been milled into the hydraulic mock up cover lid. Fig.9 shows the six gap types, for each gap type 10 thermal shock tests took place.

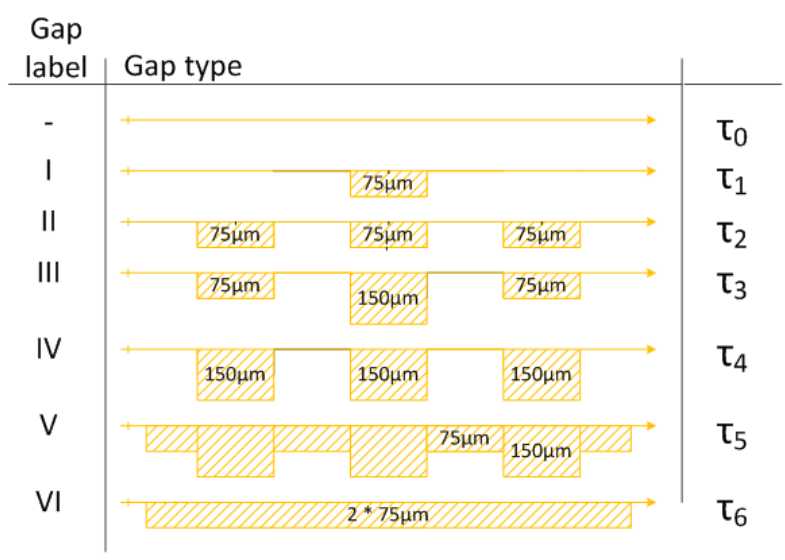

Fig.9 Artificial gap types in the thermal mock up.

Starting from gap free, a $75 \mu \mathrm{m}$ deep and $3 \mathrm{~cm}$ long slit was milled in the middle of the target element between cooling channel 1 and 2 (gap type I). Then two additional gaps with $75 \mu \mathrm{m}$ have been inserted at the beginning and the end of the target element (gap type 
II). At these positions then the gap depth was increased to $150 \mu \mathrm{m}$ (gap type III/IV). Then a $75 \mu \mathrm{m}$ gap between channel 1 and 2 over the whole length of the target element was superposed (gap type V), followed by a second gap over the whole length of the channels 3 and 4 (gap type VI).

\section{Results}

The evolution of the pressure drop during the test campaign is shown in Fig. 10.

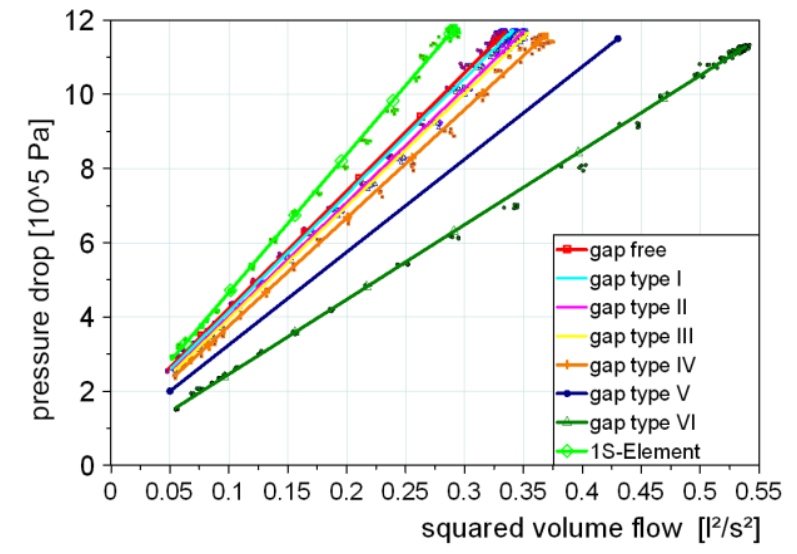

Fig.10 Pressure drop versus squared flow for the gap types defined in Fig.9.

The 1-S Elements k-value is highest as shown in Fig.10, which indicates that the new cooling structure design, shown in Fig.11, with a labyrinth sealing between the cooling channels avoids bypasses.

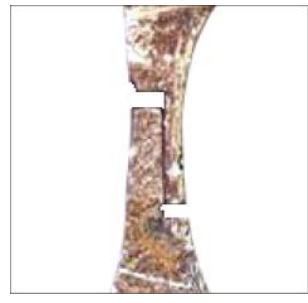

The results for the gap types I-IV are in the range of the sensors noise. For a full length gap the detection with a pressure drop measurement is feasible, the occurring pressure drop is about $10 \%$ above the theoretically expected value.

Fig.11 Crosssection of the new labyrinth sealing between cooling channels.

Table 1: Pressure drop and k-values for the 1-S pre-series target element and for the hydraulic mock up.

\begin{tabular}{rccc}
\hline Gap type & $\begin{array}{c}\Delta \mathrm{P} @ \\
8 \mathrm{~m} / \mathrm{s} \\
{\left[10^{5} \mathrm{~Pa}\right]}\end{array}$ & $\begin{array}{c}\mathrm{k} \text {-value } \\
{\left[\mathrm{bar} \mathrm{s}^{2} / \mathrm{I}^{2}\right]}\end{array}$ & $\begin{array}{c}\text { Change } \\
\text { in } \Delta \mathrm{P}[\%]\end{array}$ \\
\hline I-S & 10,2 & 37,2 & $+17,0$ \\
gap free & 9,0 & 31,8 & ----- \\
I & 8,9 & 31,3 & $-1,6$ \\
II & 8,5 & 30,7 & $-3,4$ \\
III & 8,4 & 30,6 & $-3,6$ \\
IV & 8 & 29,2 & $-8,2$ \\
V & 6,5 & 24,3 & $-23,5$ \\
VI & 5,5 & 20,4 & $-36,6$ \\
\hline
\end{tabular}

The results of the thermal shock measurements are listed in Fig.12. For the inlet side, a gap leads to a delay in the time constant, in the outlet side the cooling starts in advance and reduces the time constant. Partial gaps (I-IV) in the inlet side show no major change of $\tau$ at the outlet sided thermocouples. For the position T08, T01 and T05 the increase of $\tau$ is in the range of $35 \%$, which is close to the simulated result. Due to its position the inlet thermocouple is not affected by the bypass location, therefore tinlet is independent from the gap type.

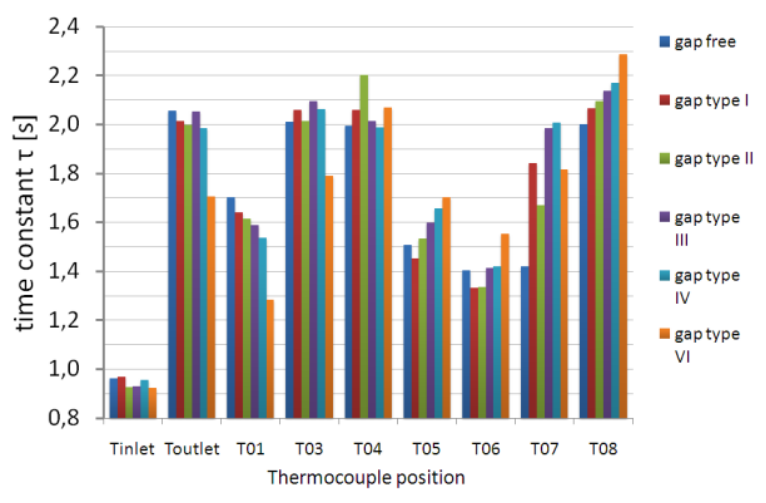

Fig.12 Resulting time constant $\tau$ for the thermocouple positions and gap types.

\section{Conclusion}

The test bed sensitivity is sufficient to detect a $75 \mu \mathrm{m}$ broad gap which spreads over the whole length of a long target element with two independent methods, but a partial opening in the cooling structure is below the detection limit which is in line with the predicted sensitivity and adequate to identify unacceptable elements. The thermal response gives a direct indication of the local flow rate, whereas with the pressure drop measurements assumptions have to be made regarded to the local coolant velocity.

Pressure drop measurements show, that the new design of the cooling structure with a labyrinth sealing leads to a $17 \%$ increase of pressure drop compared to a planar sealing which indicates that bypasses are now avoided.

The test campaign turned out that the major thread to the thermal shock method is the proper application of the thermocouples to the heat sink body. In order to be able to attach the thermocouples in a more reproducible way, an advanced mounting system is under construction, which uses pressure pistons to contact the thermocouples to the heat sink body.

\section{Acknowledgments}

This work has been performed under the grant $\mathrm{Nr}$. 03FUS0014A from the Federal Ministry of Education and Research. Only the authors are responsible for the content of this publication.

\section{References}

[1] J. Boscary et al., Fusion Engineering and Design 82 ,2007, $1634-1638$

[2] R. Tivey, Estimate of the Effect of Gaps between Adjacent Cooling Channels on $\mathrm{Cu}$ Heat Sink Temperature in W7-X Target Plates, 2008 (IPP Document) 